\title{
WHEN IS THE AUTOMORPHISM GROUP OF A VIRTUALLY POLYCYCLIC GROUP VIRTUALLY POLYCYCLIC?
}

\author{
BETTINA EICK \\ Institute of Geometry, Technische Universität Braunschweig, D-38106 Braunschweig, Germany \\ e-mail: beick@tu-bs.de
}

(Received 4 July, 2002; accepted 13 January, 2003)

\begin{abstract}
The automorphism group of a virtually polycyclic group $G$ is either virtually polycyclic or it contains a non-abelian free subgroup. We describe conditions on the structure of $G$ to decide which of the two alternatives occurs for $\operatorname{Aut}(G)$.
\end{abstract}

2000 Mathematics Subject Classification. Primary 20F16, 20F28.

1. Introduction. A group $G$ is virtually polycyclic (or polycyclic-by-finite) if it contains a polycyclic normal subgroup of finite index. The automorphism group $\operatorname{Aut}(G)$ of a virtually polycyclic group $G$ is finitely generated and embeds into $G L(n, \mathbb{Z})$ for some $n \in \mathbb{N}$. Thus, Tits' alternative yields that $\operatorname{Aut}(G)$ is virtually polycyclic or it contains a non-abelian free subgroup.

Here we consider the question: Given a virtually polycyclic group $G$, can we decide whether $\operatorname{Aut}(G)$ is virtually polycyclic without explicitly determining $\operatorname{Aut}(G)$ ?

Each virtually polycyclic group $G$ has a characteristic semisimple series; that is, a characteristic series whose factors are either finite or free abelian and rationally semisimple. We want to use the module structure of the free abelian factors in such a series to investigate $\operatorname{Aut}(G)$. We say that a free abelian subfactor $F$ of $G$ is $\mathbb{R}$ inhomogeneous if $F$ considered as a $\mathbb{Q} G$-module is a direct sum of pairwise nonisomorphic irreducible modules $F=F_{1} \oplus \ldots \oplus F_{r}$ and each $F_{i}$ either has Schur index $m_{\mathbb{Q}}\left(F_{i}\right)=1$ or $F_{i}$ is irreducible as a $\mathbb{R} G$-module. The module structure of $G$ yields the following sufficient condition for $\operatorname{Aut}(G)$ to be virtually polycyclic (see Section 3 ).

THEOREM 1.1. Let $G$ be virtually polycyclic. Then $\operatorname{Aut}(G)$ is virtually polycyclic if each free abelian factor $F$ in a characteristic semisimple series of $G$ is $\mathbb{R}$-inhomogeneous.

In certain cases, this sufficient condition is also necessary. We prove the following criterion to decide whether $\operatorname{Aut}(G)$ is virtually polycyclic (see Section 4).

THEOREM 1.2. Let $G$ be virtually polycyclic and let $F$ be a characteristic, free abelian and rationally semisimple subgroup of $G$ which has an almost complement in $G$. We denote $H=G / F$. Then $\operatorname{Aut}(G)$ is virtually polycyclic if and only if

- $F$ is $\mathbb{R}$-inhomogeneous, and

- $C_{A u t(H)}\left(H / C_{H}(F)\right)$ is virtually polycyclic.

Thus if there exists a characteristic, free abelian and rationally semisimple subgroup $F$ in $G$ which is almost complemented, then the module structure of $F$ and the automorphism group of its factor $\operatorname{Aut}(G / F)$ determine whether $\operatorname{Aut}(G)$ is virtually 
polycyclic. Note that $F$ is almost complemented if there exists a subgroup $U$ in $G$ with $U \cap F=1$ and $[G: U F]<\infty$.

Malfait and Szczepanski introduced the $\mathbb{R}$-inhomogeneous modules in [3] and used them to decide whether the automorphism group of a given Bieberbach group is virtually polycyclic. This is a special case of Theorem 1.2 as we show in Section 6 . Section 6 also includes some applications of the above Theorems and an example to demonstrate that the condition of Theorem 1.1 is not necessary in general. Section 5 gives a further analysis of $\mathbb{R}$-inhomogeneous modules.

2. Preliminaries. Each virtually polycyclic group $G$ has a characteristic series whose factors are either finite or free abelian and such a series can be determined readily without computing $\operatorname{Aut}(G)$. (See [5] for background on polycyclic groups.) We observe in the following that such a series can be refined to a characteristic semisimple series of $G$. If $F$ is a free abelian subfactor of $G$ and $K$ is a field extension of $\mathbb{Q}$, then we can consider $F_{K}=F \otimes K$ as a $K G$-module. We say that $F$ is semisimple if $F_{\mathbb{Q}}$ is semisimple as a $\mathbb{Q} G$-module. Further, the radical $\operatorname{Rad}_{\mathbb{Q} G}\left(F_{\mathbb{Q}}\right)$ is defined as the intersection of all maximal submodules of $F_{\mathbb{Q}}$.

LEMMA 2.1. Let $F$ be a characteristic, free abelian subfactor of $G$. Then the radical $R=F \cap \operatorname{Rad}_{\mathbb{Q} G}\left(F_{\mathbb{Q}}\right)$ is an Aut $(G)$-invariant subgroup of $F$ with semisimple factor $F / R$.

Proof. Aut $(G)$ acts linearly on $F_{\mathbb{Q}}$ and the maximal submodules of $F_{\mathbb{Q}}$ are permuted under this action. Hence their intersection $\operatorname{Rad}_{\mathbb{Q} G}\left(F_{\mathbb{Q}}\right)$ is $\operatorname{Aut}(G)$-invariant. Thus also $R$ is $\operatorname{Aut}(G)$-invariant. Since $F_{\mathbb{Q}}$ is finite dimensional, its radical factor is semisimple. Thus also $F / R$ is semisimple.

Lemma 2.1 yields that each virtually polycyclic group $G$ has a characteristic semisimple series which can be determined without computing $\operatorname{Aut}(G)$.

LEMMA 2.2. Let $G$ be a virtually polycyclic group and let $G=G_{1}>\ldots>G_{l+1}=1$ be a characteristic semisimple series of $G$ with $I=\left\{i \in\{1, \ldots, l\} \mid G_{i} / G_{i+1}\right.$ free abelian $\}$. Then $\operatorname{Aut}(G)$ is virtually polycyclic if and only if $\operatorname{Aut}(G)$ induces a virtually polycyclic group of automorphisms on $G_{i} / G_{i+1}$ for each $i \in I$.

Proof. Let $\operatorname{Aut}(G) \stackrel{\psi}{\longrightarrow} \prod_{i=1}^{l} \operatorname{Aut}\left(G_{i} / G_{i+1}\right) \stackrel{\varphi}{\longrightarrow} \prod_{i \in I} \operatorname{Aut}\left(G_{i} / G_{i+1}\right)$ be the natural homomorphisms. By Hall's theorem, $\operatorname{Ker}(\psi)$ is nilpotent. Since the factors $G_{i} / G_{i+1}$ for $i \notin I$ are finite, $\operatorname{Ker}(\varphi)$ is finite. Hence $\operatorname{Ker}(\psi \varphi)$ is virtually nilpotent. The image $\operatorname{Im}(\psi \varphi)$ is virtually polycyclic if and only if $\operatorname{Im}\left(\psi_{i}\right)$ is virtually polycyclic for each $i \in I$, where $\psi_{i}: \operatorname{Aut}(G) \rightarrow \operatorname{Aut}\left(G_{i} / G_{i+1}\right)$ denotes the natural action.

3. The module structure of a virtually polycyclic group. In this section we prove Theorem 1.1 and thus we obtain a sufficient condition on $G$ to have a virtually polycyclic automorphism group $\operatorname{Aut}(G)$. The proof is divided into a sequence of theorems as outlined in the following. We call a subgroup $K \leq G L(d, \mathbb{Z})$ semisimple or irreducible if the natural $\mathbb{Q} K$-module $\mathbb{Q}^{d}$ is semisimple or irreducible as a $\mathbb{Q} K$-module.

THEOREM 3.1. Let $K \leq G L(d, \mathbb{Z})$ be virtually polycyclic and semisimple. We write $C=C_{G L(d, \mathbb{Z})}(K)$ and $N=N_{G L(d, \mathbb{Z})}(K)$. Then $N / C$ is virtually abelian.

Proof. It is well-known that a semisimple, virtually polycyclic subgroup $K$ of $G L(d, \mathbb{Z})$ has a characteristic, abelian subgroup $F$ of finite index. For example, 
$Z(F i t(K))$ is of this type, see [2,6.3 and 6.5]. Let $V \cong \mathbb{Q}^{d}$ be the natural $\mathbb{Q} K$-module. Since $F$ has finite index in $K$, we have that $V$ is semisimple as a $\mathbb{Q} F$-module. Thus $V_{\mathbb{C}}$ is semisimple as a $\mathbb{C} F$-module. Since $F \unlhd N$, we can apply Lemma 1.12 of [6] and obtain that $\left[N: C_{N}(F)\right]<\infty$.

Let $U=C_{N}(F)$ and note that it suffices to prove that $U / C$ is virtually abelian. By construction, the factor $U / C$ embeds into $\operatorname{Aut}(K)$. As $F$ is central in $U$, we obtain that the image of this embedding is contained in $S=C_{A u t(K)}(F)$. The group $S$ is virtually abelian, since $C_{S}(K / F)$ is a free abelian normal subgroup of finite index in $S$. Thus $U / C$ is virtually abelian as desired.

Next, we consider the centralizer of a semisimple subgroup $K \leq G L(d, \mathbb{Z})$. The following theorem is proved in [3] for finite groups $K$. The proof is based on the investigation in [7] of the unit group of a $\mathbb{Z}$-order in the centralizer algebra of $K$ and it generalizes directly to semisimple groups $K$.

THEOREM 3.2. Let $K \leq G L(d, \mathbb{Z})$ be semisimple. Then the centralizer $C_{G L(d, \mathbb{Z})}(K)$ is virtually polycyclic if and only if the natural module for $K$ is $\mathbb{R}$-inhomogeneous.

Theorems 3.1 and 3.2 yield the following as a direct corollary.

THEOREM 3.3. Let $K \leq G L(d, \mathbb{Z})$ be virtually polycyclic and semisimple. Then the normalizer $N_{G L(d, \mathbb{Z})}(K)$ is virtually polycyclic if and only if the natural module for $K$ is $\mathbb{R}$-inhomogeneous.

Theorem 1.1 is a corollary of Theorem 3.3 and Lemma 2.2. To see this let $F$ be a free abelian factor in a characteristic semisimple series of $G$. Then $\operatorname{Aut}(F) \cong G L(d, \mathbb{Z})$ for some $d \in \mathbb{N}$ and $G$ acts as a virtually polycyclic, semisimple group $K \leq G L(d, \mathbb{Z})$ on $F$. The image of $\psi_{F}: \operatorname{Aut}(G) \rightarrow G L(d, \mathbb{Z})$ fulfills $\operatorname{Aut}(G)^{\psi_{F}} \leq N_{G L(d, \mathbb{Z})}(K)$. Thus if $F$ is $\mathbb{R}$-inhomogeneous for all free abelian factors $F$ in the given series, then $\operatorname{Aut}(G)^{\psi_{F}}$ is virtually polycyclic for all $F$ by Theorem 3.3 and $\operatorname{Aut}(G)$ is virtually polycyclic by Lemma 2.2.

4. The extension structure of a virtually polycyclic group. In this section we prove Theorem 1.2 and thus we obtain a necessary and sufficient condition for $\operatorname{Aut}(G)$ to be virtually polycyclic for certain groups $G$. This investigation is based on the extension structure of $G$. As a first step, we recall the structure of the automorphism group of an extension in the following theorem. For further details and a proof we refer to [4].

THEOREM 4.1. Let $F$ be a characteristic, free abelian subgroup of $G$ and set $H=G / F$. Let $H \rightarrow \operatorname{Aut}(F): h \mapsto \bar{h}$ be the action of $H$ on $F$ and let $P(G, F)=\{(\alpha, v) \in \operatorname{Aut}(H) \times$ $\operatorname{Aut}(F) \mid \bar{h}^{\alpha}=\bar{h}^{v}$ for all $\left.h \in H\right\}$ be the group of compatible pairs.

(a) Let $\psi: \operatorname{Aut}(G) \rightarrow \operatorname{Aut}(H) \times \operatorname{Aut}(F)$ be the natural homomorphism induced by the action of $\operatorname{Aut}(G)$ on $H$ and $F$. Then $\operatorname{Ker}(\psi)$ is abelian and $\operatorname{Im}(\psi) \leq P(G, F)$.

(b) Let $\gamma \in H^{2}(H, A)$ be a cocycle corresponding to the extension $G$ of $F$ by $H$. Then using the natural action of $P(G, F)$ on $H^{2}(H, A)$ we obtain $\operatorname{Im}(\psi)=\operatorname{Stab}_{P(G, F)}(\gamma)$.

This description of the automorphism group of an extension can be used to prove the desired Theorem 1.2 as we observe in the following.

THEOREM 4.2. Let $G$ be virtually polycyclic and let $F$ be a characteristic, free abelian and semisimple subgroup of $G$. 
(a) Suppose that $F$ has an almost complement in $G$. Then $\operatorname{Aut}(G)$ is virtually polycyclic if and only if $P(G, F)$ is virtually polycyclic.

(b) Denote $H=G / F$ and let $H \rightarrow \operatorname{Aut}(F): h \mapsto \bar{h}$ be the action of $H$ on $F$. Then $P(G, F)$ is virtually polycyclic if and only if $N_{A u t(F)}(\bar{H})$ and $C_{A u t(H)}\left(H / C_{H}(F)\right)$ are virtually polycyclic.

Proof. (a) Let $\gamma \in H^{2}(H, A)$ be a cocycle corresponding to the extension $G$ of $F$ by $H$. If $F$ has an almost complement in $G$, then $\gamma$ has finite order, see [4, 2.5]. Since $P(G, F)$ acts as a group of automorphisms on the finitely generated abelian group $H^{2}(H, F)$, we obtain that the orbit of $\gamma$ under $P(G, F)$ is finite. By Theorem $\left.4.1 \mathrm{~b}\right)$ this yields that $\operatorname{Aut}(G)$ is virtually polycyclic if and only if $P(G, F)$ is virtually polycyclic.

(b) Let $\gamma: P(G, F) \rightarrow \operatorname{Aut}(F)$ be the natural projection on the second factor and denote $C=C_{A u t(F)}(\bar{H})$ and $N=N_{A u t(F)}(\bar{H})$. By the definition of $P(G, F)$, we obtain that $C \leq \operatorname{Im}(\gamma) \leq N$. Since $F_{\mathbb{Q}}$ is semisimple, Theorem 3.1 applies and $N$ is virtually polycyclic if and only if $C$ is virtually polycyclic. In turn, we obtain that $\operatorname{Im}(\gamma)$ is virtually polycyclic if and only if $N$ is virtually polycyclic. Further, $\operatorname{Ker}(\gamma)=\{(\alpha, i d) \mid$ $\overline{h^{\alpha}}=\bar{h}$ for all $\left.h \in H\right\} \cong C_{A u t(H)}(\bar{H})$ and $\bar{H} \cong H / C_{H}(F)$.

Theorem 1.2 can now be derived directly from Theorems 3.3 and 4.2.

5. An investigation of $\mathbb{R}$-inhomogeneous modules. Let $K \leq G L(d, \mathbb{Z})$ be irreducible and denote by $V$ the natural module for $K$, that is, $V=\mathbb{Q}^{d}$ is irreducible as a $\mathbb{Q} K$-module. In this section we investigate the property of being $\mathbb{R}$-inhomogeneous in more detail. We define

$$
\begin{aligned}
& A=C_{M_{d}(\mathbb{Q})}(K) \text { its rational centralizer algebra, } \\
& B=C_{M_{d}(\mathbb{Z})}(K) \text { its integral centralizer ring, and } \\
& C=C_{G L(d, \mathbb{Z})}(K) \quad \text { its centralizer. }
\end{aligned}
$$

By Schur's lemma $A$ is a simple algebra, $B$ is a $\mathbb{Z}$-order in $A$ and $C$ is the group of units in $B$. As shown in [7] and [3] we have

$$
\begin{aligned}
& V \text { is } \mathbb{R} \text {-inhomogeneous } \\
\Leftrightarrow & A \text { is commutative or a positive definite quaternion algebra over } \mathbb{Q} \\
\Leftrightarrow & C \text { is virtually polycyclic. }
\end{aligned}
$$

We note that a basis of $A$ can be obtained by solving a system of linear equation. Also, a basis of the centre $Z(A)$ can be determined using linear algebra and thus its dimension $[Z(A): \mathbb{Q}]$ can be read off. The following lemma yields an approach to check whether $A$ is commutative or a quaternion algebra over $\mathbb{Q}$. (Compare with [1] also.)

LEMMA 5.1. Let $K \leq G L(d, \mathbb{Z})$ be irreducible and let $V$ and $A$ be as above.

(a) $\operatorname{dim}(A)=z m^{2}$, where $m=m_{\mathbb{Q}}(V)$ and $z=[Z(A): \mathbb{Q}]$.

(b) $A$ is commutative if and only if $m=1$.

(c) $A$ is a quaternion algebra over $\mathbb{Q}$ if and only if $m=2$ and $z=1$.

Proof. (a) Since $\mathbb{Q} K$ is a simple algebra, we have that $\mathbb{Q} K=M_{r}(D)$ for some division algebra $D$ of dimension $m^{2}$ over $Z(A)$. The algebra $D$ is anti-isomorphic to $A$ and thus we obtain $\operatorname{dim}(A)=z m^{2}$. 
(b) The algebra $A$ is commutative if and only if $A=Z(A)$.

(c) A quaternion algebra over $\mathbb{Q}$ has dimension 4 and $\mathbb{Q}$ as its centre by definition. Vice versa, each simple algebra of dimension 4 with centre $\mathbb{Q}$ is a quaternion algebra.

If $V_{\mathbb{R}}$ is irreducible, then $A_{\mathbb{R}}=A \otimes \mathbb{R}$ is a division algebra. By the classification of the finite-dimensional real division algebras, $A_{\mathbb{R}}$ is either $\mathbb{R}, \mathbb{C}$ or $\mathbb{H}$, the real quaternions. Thus $A$ is either commutative or a positive definite quaternion algebra. Vice versa, if $A$ is a positive definite quaternion algebra, then $A_{\mathbb{R}}$ is a division algebra and $V_{\mathbb{R}}$ is irreducible.

6. Sample applications. For practical applications of Theorem 1.2 it is useful to observe that a normal subgroup $F$ has an almost complement in $G$ if there exists a nilpotent normal subgroup $Q / F$ in $G / F$ which acts fixed-point freely on $F$. This is used in the following.

EXAMPLE 6.1. We consider the polycyclic group

$$
\begin{aligned}
G= & \langle a, b, c, d, e| b^{a}=b e, c^{a}=d, d^{a}=e, e^{a}=c d^{4}, c^{b}=c^{2} d, d^{b}=d^{2} e, e^{b}=c d^{4} e^{2}, \\
& b^{a^{-1}}=b d^{-1}, c^{a^{-1}}=c^{-4} e, d^{a^{-1}}=c, e^{a^{-1}}=d, c^{b^{-1}}=d^{-2} e, d^{b^{-1}}=c d^{4} e^{-2}, \\
& \left.e^{b^{-1}}=c^{-2} d^{-7} e^{4}(c, d, e \text { commute })\right\rangle
\end{aligned}
$$

Then $F=F i t(G)=\langle c, d, e\rangle \cong \mathbb{Z}^{3}$ is a characteristic, free abelian subgroup of $G$ with factor group $H=G / F \cong \mathbb{Z}^{2}$. The group $G$ acts on $F \cong \mathbb{Z}^{3}$ via the matrices

$$
\bar{a}=\left(\begin{array}{lll}
0 & 1 & 0 \\
0 & 0 & 1 \\
1 & 4 & 0
\end{array}\right) \quad \text { and } \quad \bar{b}=\left(\begin{array}{lll}
2 & 1 & 0 \\
0 & 2 & 1 \\
1 & 4 & 2
\end{array}\right)
$$

Since the minimal polynomials of these matrices are irreducible of degree 3 , we can readily observe that $F$ is $\mathbb{Q} G$-irreducible. This yields that $F$ is semisimple and, since $G$ acts fixed-point freely on $F$, we obtain that $F$ has an almost complement in $G$. (But $F$ is not complemented in $G$.)

Let $K=\langle\bar{a}, \bar{b}\rangle \leq G L(2, \mathbb{Z})$ be the action of $G$ on $F$ and let $A=C_{M_{3}(\mathbb{Q})}(K)$. Then $A$ is a commutative algebra of dimension 3 and hence $F$ is $\mathbb{R}$-inhomogeneous. Further, $C_{H}(F)=1$. Hence $C_{A u t(H)}\left(H / C_{H}(F)\right)=C_{A u t(H)}(H)=1$ is virtually polycyclic.

Using Theorem 1.2 we can therefore conclude that $\operatorname{Aut}(G)$ is polycyclic.

6.1. Groups with finite action. We consider the special case of Theorem 1.2 of a free abelian subgroup with finite action in the following.

THEOREM 6.2. Let $G$ be virtually polycyclic and let $F$ be a characteristic and free abelian subgroup of $G$ such that $\left[G: C_{G}(F)\right]<\infty$. Denote $H=G / F$ and suppose that $F$ has an almost complement in $G$. Then $\operatorname{Aut}(G)$ is virtually polycyclic if and only if

- $F$ is $\mathbb{R}$-inhomogeneous, and

- $\operatorname{Aut}(H)$ is virtually polycyclic.

Proof. Since $C_{G}(F)$ has finite index in $G$, we have that $G$ acts as a finite group on $F$ and thus $F$ is semisimple as a $\mathbb{Q} G$-module by Maschke's theorem. Hence Theorem 1.2 applies. Further, we note that the factor $\operatorname{Aut}(H) / C_{A u t(H)}\left(H / C_{H}(F)\right)$ embeds into the 
finite group $\operatorname{Aut}\left(G / C_{G}(F)\right)$. Hence $C_{A u t(H)}\left(H / C_{H}(F)\right)$ is virtually polycyclic if and only if $\operatorname{Aut}(H)$ is virtually polycyclic.

As a consequence of Theorem 6.2 we obtain the following theorem which has also been proved in [3]. A group $G$ is crystallographic if $\operatorname{Fit}(G)$ is free abelian of finite index in $G$ and $G$ does not contain a non-trivial normal torsion subgroup. Further, the group $G$ is a Bieberbach group if $G$ is crystallographic and torsion-free.

COROLlary 6.3 (Malfait \& Szczepanski). Let $G$ be crystallographic group and let $F=\operatorname{Fit}(G)$. Then $\operatorname{Aut}(G)$ is virtually polycyclic if and only if $F$ is $\mathbb{R}$-inhomogeneous.

Proof. Note that $F$ has finite index in $G$. Thus the trivial subgroup is an almost complement to $F$ in $G$ and $\operatorname{Aut}(G / F)$ is finite.

For a variety of explicit applications of this theorem we refer to [3].

6.2. Finitely generated nilpotent groups. Each finitely generated nilpotent group is virtually polycyclic and it has a characteristic central series whose factors are either finite or free abelian. The free abelian factors in such a series are clearly semisimple, but they are not $\mathbb{R}$-inhomogeneous unless they have dimension 1 . Hence Theorem 1.1 does not apply to this type of group. The following well-known theorem investigates the structure of the automorphism group of a finitely generated nilpotent group. We refer to [5, Chapter 1B], for background.

THEOREM 6.4. Let $G$ be a finitely generated nilpotent group and let $\psi:$ Aut $(G) \rightarrow$ $\operatorname{Aut}\left(G / G^{\prime}\right)$ be the natural action of $\operatorname{Aut}(G)$ on the characteristic abelian factor $G / G^{\prime}$. Then $\operatorname{Ker}(\psi)$ is nilpotent.

Hence the action of $\operatorname{Aut}(G)$ on the factor $G / G^{\prime}$ decides whether $\operatorname{Aut}(G)$ is virtually polycyclic or contains a non-abelian free subgroup. We give two examples, showing that both cases can occur.

EXAMPLE 6.5. Let $G=\langle a, b, c, d, e|[a, b]=c,[a, c]=d,[b, c]=e,(e, d$ central $)\rangle$ be the free nilpotent group on 2 generators with class 3 . Then $G^{\prime}=\langle c, d, e\rangle$ with $G / G^{\prime} \cong \mathbb{Z}^{2}$ and $G$ is generated by $a$ and $b$. It is straightforward to show that the maps

$$
\alpha: G \rightarrow G:\left\{\begin{array}{l}
a \mapsto a \\
b \mapsto a b
\end{array} \text { and } \beta: G \rightarrow G:\left\{\begin{array}{l}
a \mapsto a b \\
b \mapsto b
\end{array}\right.\right.
$$

induce automorphisms of $G$. Thus the image of $\psi: \operatorname{Aut}(G) \rightarrow \operatorname{Aut}\left(G / G^{\prime}\right)$ and hence also $\operatorname{Aut}(G)$ contains a non-abelian free subgroup.

A characteristic semisimple series of $G$ is given by $G>G^{\prime}>\langle d, e\rangle>1$. However, its first factor $F=G / G^{\prime}$ is not $\mathbb{R}$-inhomogeneous, since $F_{\mathbb{Q}}$ is the direct sum of two isomorphic $\mathbb{Q} G$-modules. Hence Theorem 1.1 does not apply.

EXAMPLE 6.6. Let $G=\langle a, b, c, d|[a, b]=c,[a, c]=d,[b, c]=1,(d$ central $)\rangle$. Then $G^{\prime}=\langle c, d\rangle \cong \mathbb{Z}^{2}$ and $G$ is generated by $a$ and $b$. Let $\alpha \in \operatorname{Aut}(G)$ such that $\alpha$ induces an element of $S L(2, \mathbb{Z})$ on $G / G^{\prime}$. Then $c^{\alpha} \equiv c \bmod \langle d\rangle$. Write $b^{\alpha}=a^{e} b^{f} x$ for some $e, f \in \mathbb{Z}$ and $x \in G^{\prime}$. Then we obtain

$$
1=[b, c]^{\alpha}=\left[b^{\alpha}, c^{\alpha}\right]=\left[a^{e} b^{f} x, c\right]=\left[a^{e}, c\right]^{b^{f} x}\left[b^{f}, c\right]^{x}[x, c]=\left[a^{e}, c\right]^{b^{f} x}=d^{e} .
$$


Hence $e=1$ and $b^{\alpha} \equiv b^{f} \bmod G^{\prime}$. Therefore $\operatorname{Aut}(G)$ induces an upper triangular matrix group on $\operatorname{Aut}\left(G / G^{\prime}\right)$. Thus $\operatorname{Aut}\left(G / G^{\prime}\right)$ and also $\operatorname{Aut}(G)$ are virtually polycyclic.

A characteristic semisimple series of $G$ is given by $G>G^{\prime}>\langle d\rangle>1$ and, as above, its first factor $G / G^{\prime}$ is not $\mathbb{R}$-inhomogeneous. This shows that the condition of Theorem 1.1 is not necessary to enforce that $\operatorname{Aut}(G)$ is virtually polycyclic.

\section{REFERENCES}

1. H. Brown, J. Neubüser and H. Zassenhaus, On integral groups. III: Normalizer. Math. Comp. 27 (1973), 167-182.

2. J. D. Dixon, The structure of linear groups (Van Nostrand Reinhold Company, 1971).

3. W. Malfait and A. Szczepanksi, The structure of the (outer) automorphism group of a Bieberbach group, Composito Mathematica, to appear.

4. D. J. Robinson, Applications of cohomology to the theory of groups, in Groups - St. Andrews 1981, London Math. Soc. Lecture Note Series, No. 71 (Cambridge University Press, 1981), 46-80.

5. D. Segal, Polycyclic groups (Cambridge University Press, 1983).

6. B. A. F. Wehrfritz, Infinite linear groups (Springer-Verlag, 1973).

7. H. Zassenhaus, When is the unit group of a Dedekind order solvable? Comm. Alg. 6 (1978), 1621-1627. 